\title{
The transmembrane envelope protein of HIV-I and other retroviruses modulate cytokine production and induce Treg cells Rayk Behrendt ${ }^{1}$, Viet Loan Dao Thi ${ }^{1}$, Thomas Vahlenkamp ${ }^{2}$, Reinhard Kurth ${ }^{1}$ and Joachim Denner*1
}

\author{
Address: ${ }^{1}$ Robert Koch Institute, Berlin, Germany and ${ }^{2}$ Friedrich Loeffler Institute, Greifswald-Insel Riems, Germany \\ Email: Joachim Denner* - DennerJ@rki.de \\ * Corresponding author
}

from 2006 International Meeting of The Institute of Human Virology Baltimore, USA. |7-2| November, 2006

Published: 21 December 2006

Retrovirology 2006, 3(Suppl I):S87 doi:I0.I I86/I742-4690-3-SI-S87

(C) 2006 Behrendt et al; licensee BioMed Central Ltd.

Purified retroviruses, their recombinant transmembrane envelope (TM) proteins and synthetic peptides corresponding to a highly conserved so-called immunosuppressive (isu-) domain of the TM protein have been shown to inhibit mitogen-triggered lymphocyte proliferation. The TM protein gp41 and the isu-peptide of HIV-1 have been shown to increase the expression of IL-6, IL-8, IL-10, RANTES, MCP-1, MCP-2, GRO-alpha, TNF-alpha, MIP-1alpha, MIP-1beta, MIP-3 and to decrease the expression of IL-2. Extending these studies we showed that purified Koala retrovirus (an endogenous retrovirus inducing immunodeficiencies in its host) and the TM protein of the human endogenous retrovirus HERV-K (which is highly expressed in human melanomas and teratocarcinomas) also inhibited lymphocyte proliferation and showed the same results in cytokine arrays. Furthermore, we showed for the first time that the TM protein of HERV-K and the isu-peptide of HIV-1 were able to induce CD4+CD25+ Treg cells with an increased expression of FoxP3. These data correlate with findings showing elevated IL-6, IL-10, IL-8, GRO-alpha, TNF-alpha and decreased IL-2 values as well as increased numbers of Treg in HIV infected individuals and suggest that the TM proteins may play an important role in retrovirus-induced immunopathogenesis. 